\title{
Core Community Specifications for Purchasing and Acceptance of Electron Beam Instruments
}

\author{
JOHN J. DONOVAN \\ Dept. of Chemistry, University of Oregon, donovan@uoregon.edu
}

One critical aspect of microanalysis that has been somewhat neglected from the professional community perspective are guidelines and protocols for specifying and acceptance testing of the actual instruments themselves. Generally such processes are developed independently and in parallel by various laboratories and only sporadically shared. Commercial laboratories may have some legitimate reasons for keeping such procedures secret, but university and government laboratories are under no such constraints and could greatly benefit by the sharing and pooling of ideas and suggestions. The goal here would be to present the instrument vendors with a consistent baseline for instrument specification that reflect actual real-world, "in-the-trenches" concerns for instrument performance from a user perspective.

This effort is not intended to be a "one size fits all" approach. Different laboratories often have strikingly different types of analytical problems that require specialized specification and configuration. However many of these more specific configuration and acceptance testing issues do overlap considerably from a purely instrumental performance perspective and their procedural design would benefit greatly from a community effort to pool knowledge and resources.

For example, one specific test, often termed the multiple k-ratio test, is not generally accepted by microprobe vendors as a standard measure of instrumental relative accuracy. Yet this test (where the analyst measures elemental intensities on two compositions for all spectrometers on the instrument and calculates the variance between the k-ratios obtained from each spectrometer) is the one test that actually tests the main purpose of the electron microprobe. That is to produce accurate k-ratios regardless of the spectrometer used for the measurement.

Other tests such as simple spectrometer reproducibility tests, where the reproducibility of the spectrometer intensity is measured not only between crystal flips, but also where the intensity is measured at the half intensity position are critical. These tests of the instrument's ultimate reliability under adverse conditions and are not generally tested by vendors.

These and other fundamental instrument performance parameters need to be measured and documented so the operator obtains an instrument that will produce results of a known reliability and accuracy. An example of two such tests are shown in figures 1 and 2. In the k-ratio test generally low to moderate energy lines of strong intensity in a highly absorbing matrix are preferred. Such a procedure tests, among other parameters, the effective takeoff angle of each spectrometer, the stage normal to beam, and the symmetry of analyzing crystal diffraction. The reproducibility test only requires enough intensity and/or count time to obtain reasonable statistics and tests worse case reliability. 
Andradite / Diopside, 15 keV

\begin{tabular}{|c|c|c|c|c|c|}
\hline Point & $\begin{array}{c}\text { SP1, Si Ka } \\
\text { (cps/nA) }\end{array}$ & $\begin{array}{c}\text { SP2, Si Ka } \\
\text { (cps/nA) }\end{array}$ & $\begin{array}{c}\text { SP3, Si Ka } \\
\text { (cps/nA) }\end{array}$ & $\begin{array}{c}\text { SP4, Si Ka } \\
(\mathrm{cps} / \mathrm{nA})\end{array}$ & $\begin{array}{c}\text { SP5, Si Ka } \\
(\mathrm{cps} / \mathrm{n} A)\end{array}$ \\
\hline 1 & 0.630105 & 0.632745 & 0.612295 & 0.643594 & 0.633760 \\
\hline 2 & 0.634020 & 0.635280 & 0.612271 & 0.639438 & 0.636393 \\
\hline 3 & 0.634793 & 0.636361 & 0.609293 & 0.642576 & 0.629172 \\
\hline 4 & 0.636763 & 0.635005 & 0.629825 & 0.644985 & 0.635059 \\
\hline$\ldots$ & $\ldots$ & $\ldots$ & $\ldots$ & $\ldots$ & $\ldots$ \\
\hline 17 & 0.635543 & 0.634269 & 0.625954 & 0.650216 & 0.632154 \\
\hline 18 & 0.629118 & 0.632943 & 0.665768 & 0.645899 & 0.635097 \\
\hline 19 & 0.636224 & 0.634354 & 0.669286 & 0.647104 & 0.632506 \\
\hline 20 & 0.634277 & 0.633232 & 0.656555 & 0.647342 & 0.632504 \\
\hline Average & $\mathbf{0 . 6 3 3 2 3 9}$ & $\mathbf{0 . 6 3 4 4 3 4}$ & $\mathbf{0 . 6 3 0 6 7 7}$ & $\mathbf{0 . 6 4 4 0 9 8}$ & $\mathbf{0 . 6 3 3 9 4 1}$ \\
\hline Aver Dev \% & $\mathbf{- 0 . 3 2 0 8 8 6}$ & $\mathbf{- 0 . 1 3 2 8 3 9}$ & $\mathbf{- 0 . 7 2 4 1 8 6}$ & $\mathbf{1 . 3 8 8 3 6 9}$ & $\mathbf{- 0 . 2 1 0 4 5 8}$ \\
\hline Std Dev \% & $\mathbf{0 . 0 0 2 8 9 3}$ & $\mathbf{0 . 0 0 1 2 2 5}$ & $\mathbf{0 . 0 1 9 6 1 2}$ & $\mathbf{0 . 0 0 2 7 7 9}$ & $\mathbf{0 . 0 0 2 6 7 1}$ \\
\hline
\end{tabular}

Figure 1. Multiple k-ratios measured simultaneously on all spectrometers demonstrating the overall relative accuracy of the spectrometer, crystal diffraction, beam normal and stage alignments.

\begin{tabular}{|c|c|c|c|c|}
\hline \multirow[b]{2}{*}{ Integral mode } & \multicolumn{2}{|c|}{$\begin{array}{c}\text { Spectrometer moves } \\
\text { With turret flip and back to } \mathrm{Ca}\end{array}$} & \multicolumn{2}{|c|}{$\begin{array}{c}\text { Spectrometer moves } \\
\text { With turret flip and back to Ca }\end{array}$} \\
\hline & PET - SP1 & LPET - SP2 & LPET - SP3 & PET - SP4 \\
\hline On Andradite & $\begin{array}{c}15 \mathrm{kV}-100 \\
\mathrm{nA}\end{array}$ & $15 \mathrm{kV}-30 \mathrm{nA}$ & $15 \mathrm{kV}-30 \mathrm{nA}$ & $\begin{array}{c}15 \mathrm{kV}-100 \\
\mathrm{nA}\end{array}$ \\
\hline 5um Beam Size & $\mathrm{Ca}(30 \mathrm{~s})$ & $\mathrm{Ca}(30 \mathrm{~s})$ & $\mathrm{Ca}(30 \mathrm{~s})$ & $\mathrm{Ca}(30 \mathrm{~s})$ \\
\hline $\begin{array}{l}\text { Half Peak } \\
\text { Position }\end{array}$ & 38320 & 38330 & 38330 & 38320 \\
\hline 1 & 271987 & 171354 & 383520 & 227958 \\
\hline 2 & 274750 & 169408 & 381311 & 222141 \\
\hline 3 & 271507 & 167313 & 381142 & 225177 \\
\hline & & & & \\
\hline 8 & 267135 & 172486 & 387160 & 219148 \\
\hline 9 & 269037 & 170097 & 390682 & 220483 \\
\hline 10 & 264872 & 168550 & 391850 & 225122 \\
\hline Average & 268338.7 & 170998.2 & 387370 & 222104.5 \\
\hline Std Dev & 3815.61 & 2189.08 & 3968.87 & 3315.08 \\
\hline Std Dev \% & 1.422 & 1.280 & 1.025 & 1.493 \\
\hline
\end{tabular}

Figure 2. Spectrometer reproducibility of intensities measured after repeated spectrometer motion and double crystal flips measured at the HALF intensity position demonstrating worse case reliability. 